\title{
Speed estimation of the electric-powered wheelchair by proposing the state observer method based on experimental data
}

\author{
I. M. Sollehudin ${ }^{1}$ P. M. Heerwan ${ }^{1,2}$ (D)
}

Received: 26 September 2019 / Accepted: 13 January 2020 / Published online: 20 January 2020

(c) Springer Nature Switzerland AG 2020

\begin{abstract}
The percentage of people with disabilities (PWDs) kept increasing in each year, and the demanding usage of wheelchair is also increased. This leads to more innovation which will be focused on wheelchair such as electric-powered wheelchair (EPW) and autonomous wheelchair. The innovations of the wheelchair tend to aid the PWDs with their daily routines, and it seems more acceptable in the society. When the demand for EPW is increased, the numbers of accidents among the EPW might be increased. The objective of this study is to improve the safety system of EPW by estimating the velocity of EPW. From the velocity of EPW, the autonomous system can be embedded in the EPW in order to prevent the collision. The first phase of this study is to conduct the experiment to analyse the rotational speed of the tire during straight line and obstacle avoidance. Then, the simulation is developed to design the EPW and state observer for estimating the speed of EPW. In the modelling of EPW, the input is voltage and the output is the rotational speed of the tire. Then, the modelling is validated by comparing with the experimental results. The last phase of this study is to add the state observer in the modelling to estimate the speed of EPW. In the state observer, the experimental value from the rotational tire speed will be used as a reference and compared with the simulation. The error between the experimental and simulation value is known as estimation error, and the state observer will minimize the error to get the estimated data. By estimating the velocity of the EPW, the speed of the EPW during the cornering, obstacle avoidance and straight line could be known. The focus of this study is at first and second phases, which focus on experiments to measure the rotational speed of the tire and modelling the EPW. The results shows that the error between the experimental and simulation value is below $10 \%$, and the modelling can be used for the state observer to estimate the speed of EPW.
\end{abstract}

Keywords Electric-powered wheelchair (EPW) - Obstacle avoidance $\cdot$ State observer · Dynamic behaviour · Speed estimation

\section{Introduction}

According to the statistic provided by the World Health Organization (WHO), it is estimated that $5-10 \%$ (1.3-2.6 million) of the word populations are people with disabilities (PWDs) [1]. As reported by the Department of Statistics Malaysia, the registered person with disabilities on 2017 were 453, 258 persons at the Department of Social
Welfare [2]. This number is predicted to rise along with the increasing population, lifespan and the total number of road and industrial accidents. Malaysia is expected to increase from 1.73 million (6.6\%) in 2005 to 3.8 million (11.3\%) in 2020 [3]. Thus, with the increment in the PWDs around the globe, the numbers of the wheelchair user also will be rising in each year according to the statistics.

$\triangle$ P. M. Heerwan, mheerwan@ump.edu.my; I. M. Sollehudin, sollehudin92@gmail.com | ${ }^{1}$ Faculty of Mechanical and Automotive Engineering Technology, Universiti Malaysia Pahang, 26600 Pekan, Pahang, Malaysia. ${ }^{2}$ Automotive Engineering Centre, Universiti Malaysia Pahang, 26600 Pekan, Pahang, Malaysia. 
The development of the manual wheelchair in recent years improves the daily routine of the PWDs. The implications of the propulsion of manual wheelchairs by the user also can be severe if the user does not improve the way on how he or she propels the wheelchair [4]. The study also shows that the manual wheelchair user suffers the long-term pain on musculoskeletal part of the body [5]. Even though there are many development plans to aid the manual wheelchair, the long-term injury still occurs [6]. The development of the wheelchair also improved nowadays, whereas the wheelchair is also solely developed for the specific impairment of the users [7]. The movement for the patients that paralysed from upper to lower body will be extremely difficult for their daily routine. Some researchers develop smart wheelchair that recognize the voice and the body gesture to navigate the wheelchair $[8,9]$. The development of the self-navigated wheelchair also increases and mostly under development [10]. Most of the wheelchair focused on the path planning and the methods that could detect the obstacle more precisely. This paper aims to estimate the velocity of the electricpowered wheelchair (EPW) during the obstacle avoidance. From a research in a rehabilitation centre for the elderly, the speed that is recorded for the EPW user is $2.7 \mathrm{~m} / \mathrm{s}$ [11].

The speed recorded from the rehabilitation is for the straight line and not for the obstacle avoidance. When one of the EPW tire speed is higher than the other, the EPW turns in an arc towards the slower wheel result in turning condition. The speed of the EPW lies between the speed of right and left tires. Thus, the state observer method is proposed in determining the EPW, speed whereas the EPW's speed is estimated from the simulation [12]. In order to gain the EPW speed, the data from the experiment are also required for simulation and by using the reliable sensors to gain the accurate and precise data [13]. With the state observer method, it could estimate the parameters such as the state recovery and the disturbances in an area [14]. This proposed method could determine and analyse the speed of the EPW during the obstacle avoidance. Furthermore, the application of the state observer also can be seen in detecting the fluctuation of torque on brushless $\mathrm{DC}$ motor (BLDCM) which is not reachable for the sensors to measure [15].

\section{Experimental procedures}

This experiment was conducted to acquire the data of the longitudinal and lateral acceleration and the velocity of the both tires. The user in this experiment is an adult male with one-year experience using the EPW. In order to obtain the reliable data, the EPW will be maneuvered into

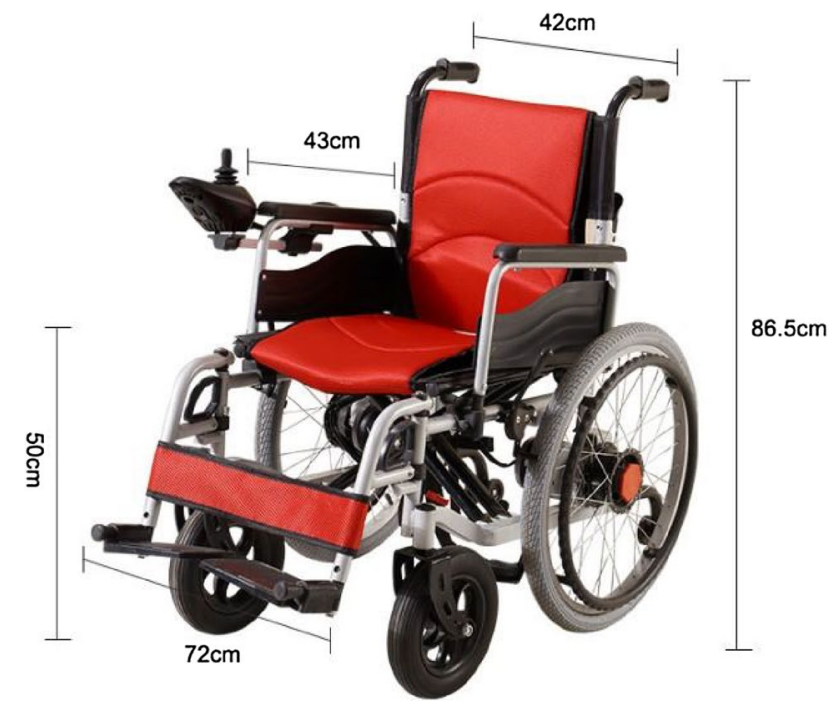

Fig. 1 Electric-powered wheelchair

Table 1 Electric-powered wheelchair specifications

\begin{tabular}{ll}
\hline Specifications & Capabilities \\
\hline Motor power & $250 \mathrm{~W}$ per motor \\
Climbing ability & $<13^{\circ}$ \\
Battery & $24 \mathrm{~V}, 12 \mathrm{~A}$ \\
Battery endurance & $10-15 \mathrm{~km}$ \\
Speed mode & 6 Speed adjustment \\
Brand & Jikang \\
Model & Tw100 \\
Wheel size & 10 Inch (Front), 22 Inch (Rear) \\
Weight & $34 \mathrm{~kg}$ (Without battery) \\
User & Elder and disables \\
\hline
\end{tabular}

the situation that is same as an autonomous wheelchair would performed.

\subsection{Electric-powered wheelchair}

Figure 1 shows the electric-powered wheelchair (EPW) that has been used in the experiment. This EPW consists of two electric motors, joystick to control the movement and LiPo battery.

Figure 1 also shows the dimensions of the EPW that are being used during the experiment, while Table 1 shows the detailed specifications of the EPW.

\subsection{Experimental setup}

In the experiment, the road surface is dry and the experiment is conducted in the close environment (inside the laboratory). The setup for the experiment was divided into 
two conditions: the straight line without an obstacle and when avoiding an obstacle. A static obstacle which is a box is used in this experiment, and the user needs to avoid this obstacle. This situation is same as in the occasion of the autonomous wheelchair.

The parameters such as velocity and distance are being kept constant in order to preserve the data, and all of the experiments are conducted five times in order to yield the results in the consistency manners. All the possible interferences must be planned well in order to give a consistency of the data for the experiment.

\subsection{Sensors}

For this experiment, sensors that are being used to collect the speed data are the rotary encoder. The lateral and longitudinal acceleration is acquired by using the gyroscope. The gyroscope is a sensor that directly acquires the lateral and longitudinal acceleration data, while the rotary encoder, some mathematical equation, needs to be used in order to acquire speed data. The rotary encoder writes the signals in the form of pulse. This pulse needs to be translated into the speed, and some equations need to be developed.

Calculations need to be developed in order to determine the velocity of the both tires. The equation determines the velocity of the tires as shown below, the EP, Encoder Pulses and $r$, radius of the tire.

Encoder $=\frac{E P}{\text { Pulse per Revolution }} \times \frac{60 \mathrm{~s}}{1 \mathrm{~min}}$

$\mathrm{EP}=\mathrm{EP}_{n}-\mathrm{EP}_{n-1}$

Equation (1) shows the translation of rotary encoder signals into mathematical formula. This equation will be used to translate into velocity.

Velocity $=\frac{\text { Encoder }}{\text { Sampling Time }} \times 2 \pi r$

The signal input of Eq. (1) will be taken into Eq. (3) to yield the velocity output. The sensors are attached on both tires in order to acquire both velocities of tires (Fig. 2).

\subsection{Modelling of DC motor}

After the experiment, the next procedure is to model the EPW into the MATLAB and where the DC motor and kinematic equation of the EPW are involved. The equation shown derives the Kirchhoff's law from the DC motor circuit, induction, $L$, voltage, $V$, resistance, $R$, current, $i$, constant, $K$, and angular velocity.
Fig. 2 Illustration of the experimental setup
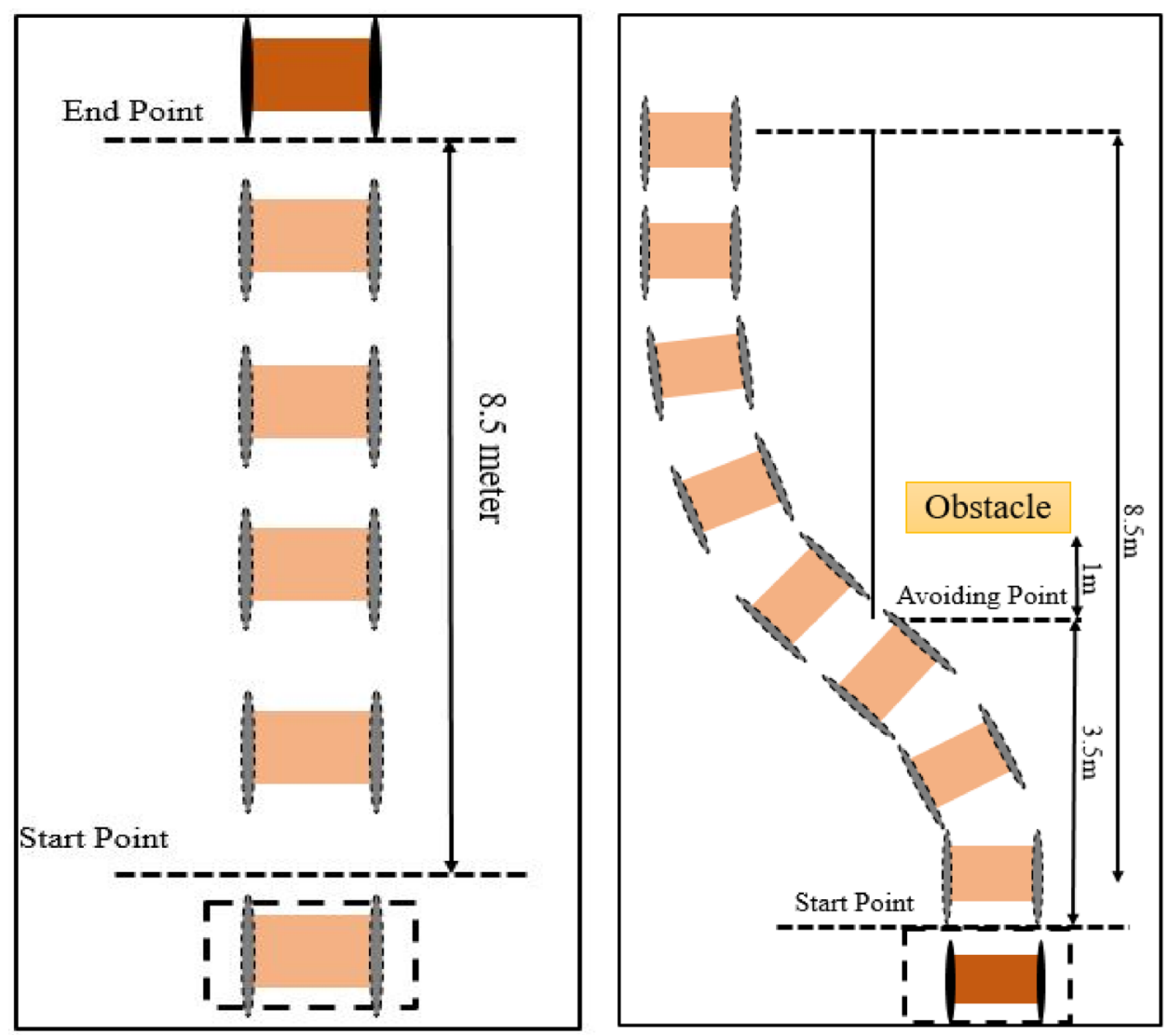
$L \frac{\mathrm{d} i}{\mathrm{~d} t}=V-R i-K_{e} \dot{\vartheta}$

$J \frac{\mathrm{d} \ddot{\vartheta}}{\mathrm{d} t^{2}}=T_{m}-T_{F}-b \dot{\vartheta}$

The Newton's law is being applied in the following equations based on the mechanical properties of the DC motor with the moment inertia, $J$, torque motor, $T_{m}$, friction motor, $T_{F}$, and motor bearing, $b$.

\subsection{Modelling of wheelchair}

Modelling the dynamics behaviour of the wheelchair also deals with the mathematical expressions. From the Newton's second law of motion, the summation of longitudinal forces, $F_{x}$ equals to the mass, $m$, multiplied by the acceleration, $a$.

$\sum F_{x}=m a$

The equation of the longitudinal force is shown below where mass is denoted as $m$, gravitational force, $g$, and coefficient of friction, $\mu$.

$F_{X}=(m \cdot g) \cdot \mu$

The equations above are being applied in determining the longitudinal force for each tire of the wheelchair. The equations are then being used to define the speed of the tires and EPW. Figure 3 shows the block diagram on how the equations being applied in order to define the longitudinal speed of the EPW.

\section{State observer}

The modelling of the state observer is to estimate the velocity of the EPW during the obstacle avoidance. From the experiments, the data that could be retrieved from the sensors are the speed of tires. Implementing the state observer method, the estimated value of EPW could be determined by using the experimental data into the simulation.

\subsection{Modelling the state observer}

State observer will be modelled in the MATLAB in which the EPW also will be modelled. The modelling of the EPW will be accordingly to the mathematical equation in order to yield the velocity output for both left and right tires. The longitudinal and lateral acceleration will be utilized to determine estimation value of the EPW during the obstacle avoidance.

Figure 4 shows the model of the state observer; it is only the general concept of the state observer. The measured value is the experimental value obtained from the
Fig. 3 Block diagram determining the EPW speed

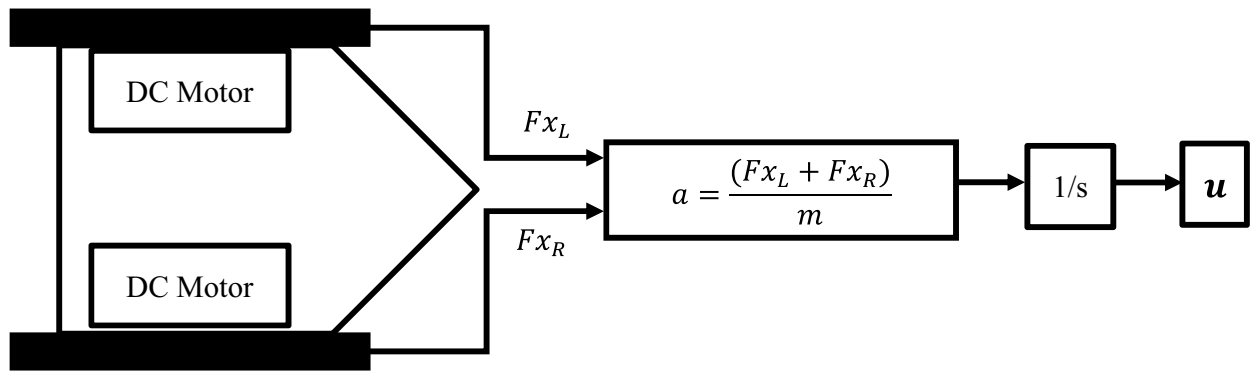

Fig. 4 Block diagrams for fullstate observer

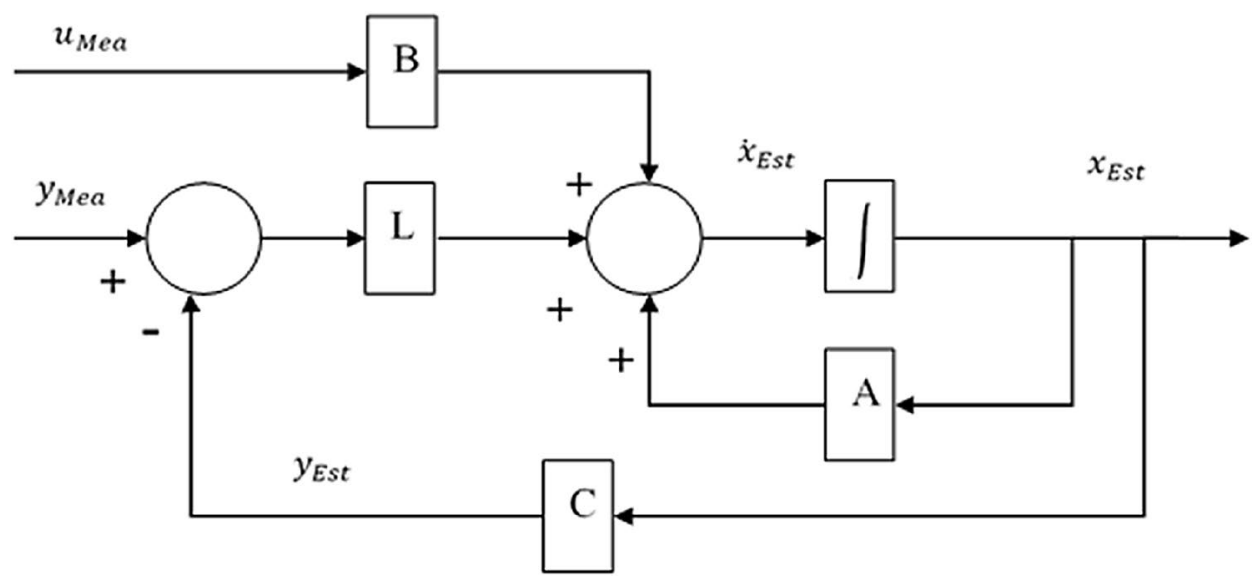


experimental procedure as being mentioned earlier. Meanwhile, the estimated value is acquired from the simulation based on the experimental results.

Equations below show the state space equation for experimental and simulation that are noted as the measured and estimated value, respectively. Also, it can be noted as the observer model. $L$ is the $n \times m$ gain matrix for this model. The state space for Eq. (8) is accordingly to the measured state space but being replaced with the estimated state variables in Eq. (9).

$\dot{x}_{\text {Mea }}=A x_{\text {Mea }}+B u_{\text {Mea }}$

$y_{\text {Mea }}=C x_{\text {Mea }}+D u_{\text {Mea }}$

$\dot{x}_{\mathrm{Est}}=A x_{\mathrm{Est}}+B u_{\mathrm{Mea}}+L\left[y_{\mathrm{Mea}}+y_{\mathrm{Est}}\right]$

$y_{\mathrm{Est}}=C x_{\mathrm{Est}}+D u_{\mathrm{Mea}}$

$\dot{x}_{\mathrm{Est}}=A x_{\mathrm{Est}}+B u_{\mathrm{Mea}}+L y_{\mathrm{Mea}}-L C x_{\mathrm{Est}}-L D u_{\mathrm{Mes}}$

$=(A-L C) x_{\mathrm{ESt}}+(B-L D) u_{\mathrm{Mea}}+L y_{\mathrm{Mea}}$

By substituting the $y_{\text {Est }}$ into the observer's state equation, the alternative form for the equation is yielded.

Figure 3 shows the block diagram with all the state equation and significantly shows the full-state observer model.

\subsection{Estimation error}

Estimating the error is another method that being used in the state observer in order to yield an estimation of true state $x_{\text {Est }}$. The estimation error purposely to reduce the error exists during the initial time. The error will gradually reduce proportionally with time.

$e=x_{\text {Mea }}+x_{\text {Est }}$

Equation above shows the definition of the error estimation.

\section{Results}

These are the results that obtained from the experiment on the EPW. These results consist of two separated experiment that is done by the user by manoeuvring the EPW with the passive control and not the autonomous system. The first experiment was during the straight line, and the second experiment was the obstacle avoidance.

\subsection{Tire velocity on straight line}

Figure 5 shows the result of right, left tires and the average of the right and left tires against time. The experiment conducted to measure the velocity of both tires during the straight line and theoretically the both velocities for left and right tires should almost be equal as shown in Fig. 5

\subsection{EPW longitudinal acceleration}

Figure 6 shows the acceleration of EPW and average acceleration of the EPW against time. The lateral acceleration is absent due to the experiment done on the straight line; thus, the lateral acceleration is assumed to be zero. The trend can be said almost consistent due to the experiment done in the controlled environment. The acceleration reached almost zero acceleration as the factor of the EPW reach the constant velocity on the straight line.

\subsection{Tire velocity on obstacle avoidance}

The experiment is then conducted for the obstacle avoidance.

The distance for the obstacle avoidance experiment is also defined before the experiment conducted by assuming all the circumstances such as the distance of the EPW before impact with the obstacle and preventing the user colliding with the obstacle.

Figure 7 shows the result acquired from the obstacle avoidance experiment. From Figs. 5 and 7, the results of the speed of the tires are much more different comparatively due to the presence of the obstacle. The differences between these two experiments can be seen from the trend of the results of the obstacle avoidance. As the EPW reach the obstacle avoidance distance, the velocity for both tires changes drastically as shown in Fig. 7. This is due to the rotational speed difference in right and left tires of the EPW.

In order to navigating away from the obstacle, the user will manoeuvre the EPW away from the obstacle but the path of the EPW will be corrected again since the EPW is on the right track and reaches the finish line.

In order to give clear view of the difference in rotational speed of the right and left tires, the results of the right and left tires are being average as shown in Fig. 6. From the averaged velocity of right and left tires, the differences in velocity of both right and left tires change during the early of $6 \mathrm{~s}$. This is where the EPW encounter the obstacle, and the tire velocity once again changed at $7 \mathrm{~s}$ where the user that manoeuvre the EPW is correcting the path of the EPW.

\subsection{EPW longitudinal and lateral acceleration on obstacle avoidance}

From the experiment of the EPW on the straight line, the EPW lateral acceleration of the EPW is zero due to the 

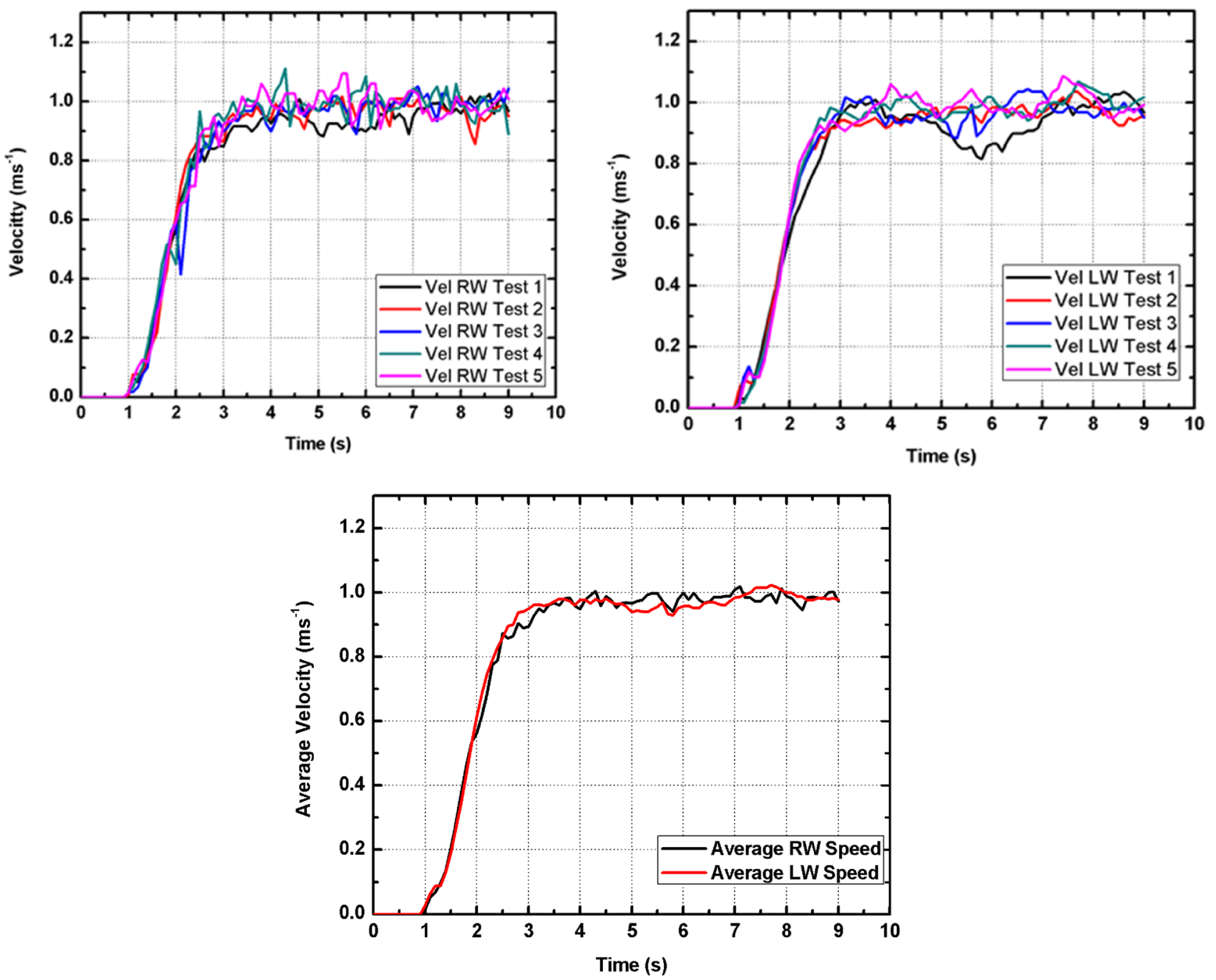

Fig. 5 Velocity of right tire against time, velocity of left tire against time and average velocity of right and left tires against time
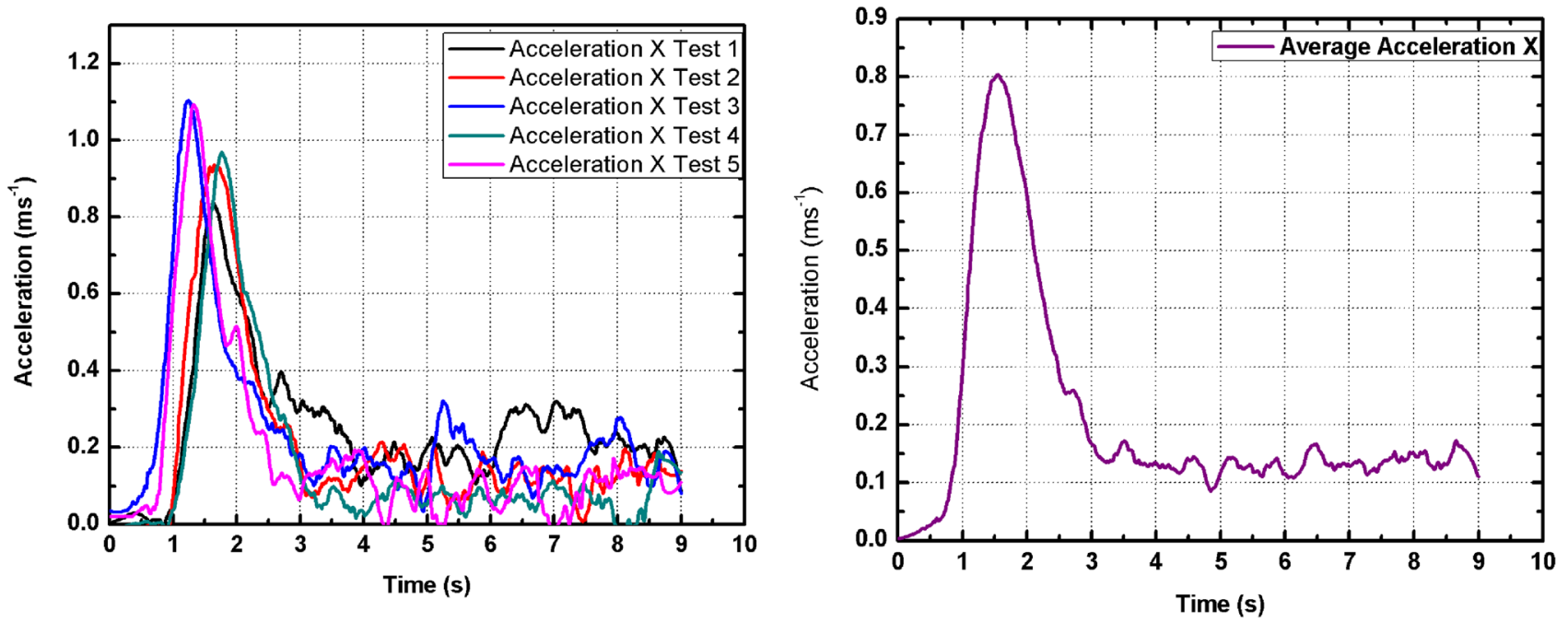

Fig. 6 Acceleration of EPW against time and average acceleration of EPW against time 



Fig. 7 Velocity of right tire against time, velocity of left tire against time and average velocity of right and left tires against time

experiment conducted on straight line, and the lateral acceleration presence indicates almost zero acceleration.

Figure 8 presents the results of the longitudinal and lateral acceleration of the EPW against time. From the lateral acceleration, the EPW clearly can be seen is avoiding the obstacle from the data acquired. The changes in the lateral acceleration on $3 \mathrm{~s}$ indicate that the EPW is avoiding the obstacle, while the lateral acceleration changes once again indicating that the EPW is correcting the navigational path.

The longitudinal acceleration of the EPW is almost unaffected due to the speed during the obstacle avoidance which is almost equal as the straight line experiment even though there is slight drop of the longitudinal acceleration during the obstacle avoidance.
Figure 8 also shows the averaged longitudinal and lateral acceleration which gives a clear view on how the experiment of the EPW during the obstacle avoidance is happened, while the averaged lateral acceleration clearly shows how the EPW mitigates the obstacle.

\section{Conclusions}

Based on the experiment of the EPW, the rotary encoder sensors are being used to determine the changes of speed of the EPW tires during the obstacle avoidance. The result shown indicates the rate of change between right and left tires. The gyroscope sensors are not only 

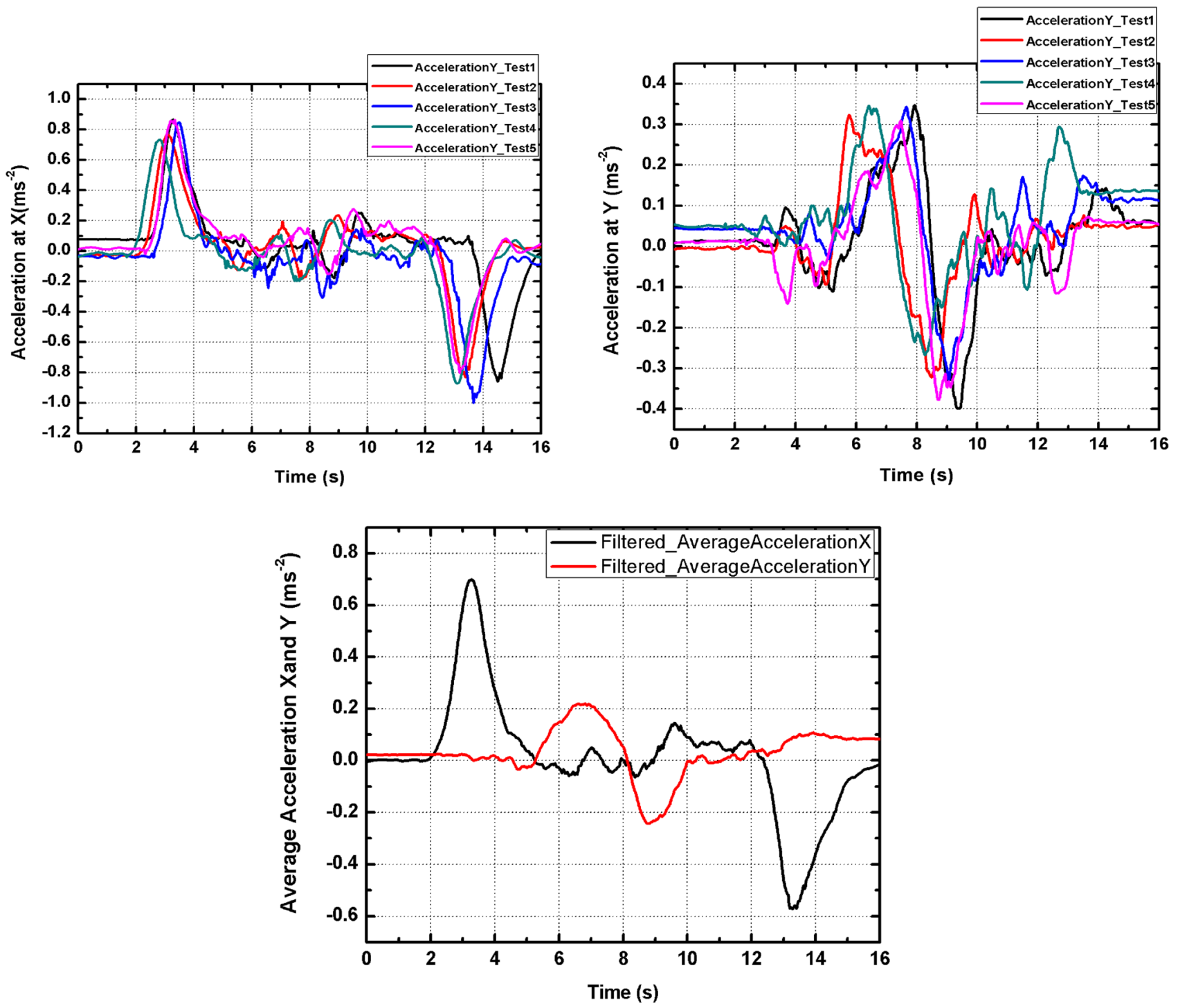

Fig. 8 Longitudinal acceleration against time, lateral acceleration against time and average longitudinal and lateral acceleration against time

capable in measuring the longitudinal and lateral acceleration, but also show the direction of the EPW during the obstacle from the lateral acceleration.

Determining the estimated longitudinal speed of the EPW will be reliable only on the straight line but not for the obstacle avoidance. When involved in the obstacle avoidance experiment, both right and left tire velocities change significantly, and the speed of the EPW could be varied during the occurrence. Thus, the longitudinal and lateral acceleration data from the experiment can be used to estimate the speed of the EPW during the obstacle avoidance. The estimated speed of the EPW throughout the obstacle avoidance could be determined.
Acknowledgements The authors would like to thank Universiti Malaysia Pahang (www.ump.edu.my) for the financial support through the UMP internal Grant RDU190321.

\section{Compliance with ethical standards}

Conflict of interest The authors declare that they have no competing interests. 


\section{References}

1. Rahim AA, Samad NAA (2010) Accessible built environment for the elderly and disabled in Malaysia: hotels as case studies. J Constr Dev Ctries 15(2):1-21

2. Mahidin MU (2018) Registration of persons with disabilities (PWD). Social Statistics Bulletin Malaysia 2018, November 29, 2018

3. Onken SJ, Craig CM, Ridgway P, Ralph RO, Cook JA (2007) An analysis of the definitions and elements of recovery: a review of the literature. Psychiatr Rehabil J 31(1):9-22

4. Regazzoni D, Vitali A, Rizzi C, Colombo Zefinetti F (2019) Motion capture and data elaboration to analyse wheelchair set-up and users' performance. In: ASME 2018 International Mechanical Engineering Congress and Exposition. American Society of Mechanical Engineers Digital Collection

5. Finley MA, Euiler E (2019) Association of musculoskeletal pain, fear-avoidance factors, and quality of life in active manual wheelchair users with SCl: a pilot study. J Spinal Cord Med 1-8. https://www.tandfonline.com/action/doSearch?AllField=Marga ret+A.+Finley

6. Vincent C, Gagnon DH, Dumont F, ADMI group (2019) Pain, fatigue, function and participation among long-term manual wheelchair users partnered with a mobility service dog. Disabil Rehabil Assist Technol 14(2):99-108

7. Dey P, Hasan MM, Mostofa S, Rana Al (2019) Smart wheelchair integrating head gesture navigation. In: 2019 International conference on robotics, electrical and signal processing techniques (ICREST), IEEE, pp 329-334

8. Joseph C, Aswin S, Prasad JS (2019) Voice and gesture controlled wheelchair. In: 2019 3rd International conference on computing methodologies and communication (ICCMC), IEEE, pp. 29-34
9. Bhuyain MF, Shawon MA-UK, Sakib N, Faruk T, Islam MK, Salim KM (2019) Design and development of an EOG-based system to control electric wheelchair for people suffering from quadriplegia or quadriparesis. In: 2019 International conference on robotics, electrical and signal processing techniques (ICREST), IEEE, pp 460-465

10. Röfer T, Lankenau A (2000) Architecture and applications of the Bremen Autonomous Wheelchair. Inf Sci 126(1-4):1-20

11. Cooper RA et al (2002) Driving characteristics of electric-powered wheelchair users: how far, fast, and often do people drive? Arch Phys Med Rehabil 83(2):250-255

12. Bersani M, Vignati M, Mentasti S, Arrigoni S, Cheli F (2019) Vehicle state estimation based on Kalman filters. In: 2019 AEIT International conference of electrical and electronic technologies for automotive (AEIT AUTOMOTIVE), IEEE, pp 1-6

13. Hsu L-Y, Chen T-L (2008) Vehicle full-state estimation and prediction system using state observers. IEEE Trans Veh Technol 58(6):2651-2662

14. Liu L, Wang D, Peng Z (2019) State recovery and disturbance estimation of unmanned surface vehicles based on nonlinear extended state observers. Ocean Eng 171:625-632

15. You S, Gil J, Kim W (2019) Extended state observer based robust position tracking control for DC motor with external disturbance and system uncertainties. J Electr Eng Technol 14:1637-1646. https://doi.org/10.1007/s42835-019-00162-5

Publisher's Note Springer Nature remains neutral with regard to jurisdictional claims in published maps and institutional affiliations. 